\title{
Genética: aportes al pensamiento evolutivo
}

\author{
Ana Beatriz Mafla \\ Escuela de Ciencias Biológicas de la Pontificia Universidad Católica del Ecuador. \\ amafla@puce.edu.ec
}

En la evaluación de los aportes de la Genética a la teoría evolutiva haré referencia a tres períodos de aproximadamente 50 años cada uno, más o menos sobrelapados entre ellos. El primero, desde 1859 a 1909 , se caracteriza porque la publicación de la obra El Origen de las especies trajo consigo una revolución en el pensamiento y se da un vuelco hacia el materialismo filosófico. El segundo, desde 1909 a 1959, incluye el redescubrimiento de las leyes mendelianas, el origen y desarrollo de la Genética como ciencia y el nacimiento de la síntesis moderna. Y el tercero, desde 1959 a 2009, comprende el auge de la biología molecular. En este periodo somos testigos de los debates entre neutralistas y seleccionistas, así como de la pretendida urgencia de construir una nueva síntesis.

El primer período, que abarca los primeros cincuenta años desde la publicación de El Origen, lo podemos caracterizar como signado por la ausencia de la Genética. Sin embargo, de los 15 capítulos del libro, los cinco primeros ${ }^{1}$ se destinan a la VARIACIÓN y a la SELEC-
CIÓN dejando sentada la idea de que estos son los dos fenómenos nucleares de la teoría de "La descendencia con modificación". En los siguientes cinco capítulos, ${ }^{2}$ Darwin presenta los puntos debatibles de su teoría y asume el desconocimiento de las leyes de la herencia y la variación. A pesar de la ausencia de una teoría de la herencia fuerte, Darwin propugnó la teoría de la descendencia con modificación como la responsable de las afinidades entre los seres orgánicos, incluido el ser humano; y en el capítulo 14 claramente declara: "El sistema natural es un ordenamiento genealógico, en el que se expresan los grados de diferencia adquiridos, por las variedades, especies, géneros, familias, etc.". Desde entonces, esta idea es el telón de fondo de todas las investigaciones en el campo de la Biología; de tal manera que la Evolución como teoría adquiere la categoría de paradigma científico. La revolución darwiniana fue considerada peligrosa desde su inicio, pues la idea central de ella es el cambio; frente a la idea de fijismo hay clara

\footnotetext{
1. La variación en estado doméstico. 2. La variación en la naturaleza. 3. La lucha por la existencia.

4. Selección natural o la supervivencia de los más aptos. 5. Las leyes de la variación.

6. Dificultades de la teoría. 7. Objeciones diversas a la teoría de la selección natural. 8. Instinto. 9. Hibridismo. 10. Imperfección de los datos geológicos.
} 
oposición a la de inmutabilidad de las especies, ... del hombre, ... de la sociedad, por ello fue combatida no sólo en el medio académico sino en todo el entorno social.

El segundo período (1909 a 1959) corresponde a los aportes de la Genética a la Teoría evolutiva; en él, visualizo dos momentos: el primero referido al redescubrimiento de las leyes mendelianas y sus efectos: el Mendelismo permitió abandonar la teoría de la herencia mezclada y reemplazarla con la teoría de la herencia particulada; aunque paradójicamente se disminuyó el prestigio del darwinismo a favor del mutacionismo; el dogma aceptado acerca de los mecanismos de la evolución expresado en The Scientific Basis of Evolution de T. H. Morgan (1932), resalta dos ideas centrales: 1) la Selección Natural no es más que la vía de purificación del plasma germinal; y 2) la Mutación es el único factor significativo de cambios evolutivos. En el segundo momento donde domina la teoría de la herencia particulada, hay dos hechos importantes: uno es la restauración del prestigio de la teoría darwiniana y el reconocimiento de la Selección Natural como factor principal de la evolución; hecho que se debe al nacimiento de la genética de poblaciones, con los trabajos de Chetverikov, S. S. , R. A. Fisher, S. Wright y J. B. S. Haldane . El segundo hecho es el surgimiento de la Síntesis Moderna entre los años 1930 a 1950. Esta síntesis se consolida por los aportes de tres disciplinas: Genética, Sistemática y Paleontología y tres personajes: Theodosius Dobzhansky, Ernst Mayr, George Simpson, quienes independientemente adoptaron el pensamiento poblacional y lo desarrollaron en sus obras ; la síntesis se consolida durante el Congreso de Princeton en 1947, donde también se decide la publicación periódica de la revista Evolution y los tres científicos antes mencionados hacen el compromiso de profundizar sus ideas; fruto de este compromiso son las obras: The Major Features of Evolution de G. Simpson (1953); Animal Species and Evolution de E. Mayr (1963) y Genetics of Evolutionary Process de T. Dobzhansky (1970). Con lo dicho, el segundo período se puede caracterizar porque en él hay una consolidación de la teoría Darwiniana asistida por el Mendelismo y a la que identificamos como Neodarwinismo.

El tercer período está marcado por el desarrollo de la biología molecular y la tecnología del ADN recombinante que conduce a la segunda revolución importante en las Ciencias Biológicas. El uso de las técnicas electroforéticas posibilitó analizar no sólo la variación genética de las poblaciones sino también describir el flujo génico entre ellas o estudiar la Selección Natural e inferir las primeras filogenias moleculares. En el primer caso, los estudios de variación genética de las poblaciones permitieron resolver el debate entre Clasicistas y Balancistas a favor de la teoría del Balance. Las técnicas moleculares, así mismo, determinan nuevas propuestas como las de Neutralistas como M. Kimura, T. Ohta, T. Maruyama, M. Nei y Seleccionistas como Carson, R. Lewontin, Wallace, D. Brncic, Cordeiro, herederos de las escuelas Clasicista y Balancista, respectivamente; y también inducen a nuevos cuestionamientos y polémicas 
como las de Gradualistas y Saltacionistas. Las nuevas tecnologías en Biología Molecular, entonces, aparte de abonar el campo de discusión en Teoría Evolutiva, posibilitan la manipulación de la vida $\mathrm{y}$ nos hacen reflexionar sobre el futuro de la Humanidad; de tal forma que en la toma de decisiones dentro del escenario global del siglo XXI queda abierta la pregunta: ¿Quo vadis homo?

\section{BIBLIOGRAFÍA}

Blanc, Marcel. 1982. Las teorías de la Evolución hoy. Mundo Científico, $\mathrm{N}^{\circ}$ 12: 288303.

Darwin, Charles. 1859. El Origen de las Especies. Traducción 1973. Editorial Albatros, Buenos Aires. 339 pp.

Futuyma, Douglas J. 1986. Evolutionary Biology, second edition. Sinauer Associates, Inc. Publishers, Sunderland, Massachusetts. $600 \mathrm{pp}$.

Mayr, Ernst. 1992. Una larga controversia: Darwin y el Darwinismo. Crítica, Grijalvo Comercial, S. A., Barcelona. 209 pp.

Mayr, Ernst. 2001. What Evolution Is, Basic Books, New York. 318 pp. 